\title{
Diseño y desarrollo de un ciclo de mejora en el aula para la enseñanza-aprendizaje de la asignatura de Química Orgánica I en el grado en Farmacia
}

\section{Design and development of a cycle of improvement in the classroom for the teaching- learning of the subject of Organic Chemistry I in the degree in Pharmacy}

VICTORIA VALDIVIA GIMÉNEZ

ORCID: https://orcid.org/0000-0003-3534-7074

Universidad de Sevilla

Departamento de Química Orgánica y

Farmacéutica

vvaldivia@us.es

DOI: http://dx.doi.org/10.12795/9788447231003.083

Pp.: 1791-1814 


\section{Descripción del contexto}

El ciclo de mejora en el aula (en adelante CIMA) recogido en este trabajo se realizó en el contexto de la asignatura de Química Orgánica I de primero del Grado en Farmacia. Se trata de una asignatura que tradicionalmente se ha etiquetado como dificil y poco interesante entre los alumnos de Farmacia, de hecho, una mayoría de ellos se inscriben en clases particulares antes incluso de matricularse en el curso oficial, dejándose llevar por los rumores que desde el inicio de la carrera circulan entre los estudiantes. Además, se ha extendido entre los miembros de la facultad la equivocada creencia de que ni siquiera es una asignatura esencial para ejercer la profesión farmacéutica en sus numerosas facetas. Todo esto, sumado a la necesidad de generar en los estudiantes capacidad de abstracción y visión tridimensional fundamentales para la comprensión de la asignatura, han constituido los principales obstáculos que se han pretendido salvar a través del nuevo modelo de enseñanza-aprendizaje que aquí se presenta.

Por otro lado, dada la situación sanitaria en la que actualmente nos encontramos, los alumnos matriculados en el grupo, que eran alrededor de 80 , se encontraban formando subgrupos de unos 20 miembros, de modo que cada semana acudía a clase sólo uno de estos subgrupos y el resto, seguían las sesiones on line a través de Blackboard Collaborate, la herramienta de la enseñanza virtual de la Universidad de Sevilla para la docencia on line. Las condiciones de trabajo eran, por lo tanto, relativamente adecuadas, pero a esto se sumaba la obligatoriedad de mantener una distancia mínima de seguridad de alrededor de 2 metros que dificultaba considerablemente el trabajo en grupo de los alumnos asistentes.

Ciclos de Mejora en el Aula (2020). Experiencias de Innovación Docente de la US Esta obra se distribuye con la licencia Creative Commons 


\section{Diseño previo del CIMA}

\section{Principios didácticos}

El principal principio didáctico que se tuvo en cuenta se basó en la experiencia narrada por Don Finkel en su libro Dar clase con la boca cerrada (Finkel, 2008). En el capítulo 6 del citado libro, el autor hace referencia a que formular problemas centrados y directos e invitar a los alumnos a trabajar en ellos en grupos pequeños, puede ser una alternativa a la tradicional clase magistral.

Para ser capaz de formular los problemas y/o preguntas que estructuren el contenido de un tema, el docente, debe realizar un trabajo previo de elaboración de un mapa de contenidos que refleje las interacciones más potentes entre los llamados contenidos estructurantes, así como los principales valores, procedimientos y datos que se relacionan con ellos. El mapa de contenidos ayuda al docente a pensar y diseñar preguntas para cada contenido estructurante (pregunta estructurante). Después de plantear estas preguntas, se deben diseñar actividades encadenadas como ayuda para organizar y facilitar sus razonamientos. Estas actividades se consideran una guía para resolver cada pregunta pero sin proporcionar una respuesta. Para Finkel (2008), este es el proceso que conduce a la comprensión y, de aquí, al conocimiento. Las actividades encadenadas, no deben narrar directamente ni anunciar los resultados, sino que tienen que animar a los alumnos a hacer descubrimientos y pensar por ellos mismos, para encontrar sus resultados y posteriormente contrastarlos con los de los demás grupos y con la opinión del docente y de esta forma generar un aprendizaje duradero. Siguiendo con este autor, consideramos que elaborar las actividades es un arte y para hacerlo bien, el profesor debe conocer bien a los alumnos y la materia que imparte, ya que cada

Ciclos de Mejora en el Aula (2020). Experiencias de Innovación Docente de la US Esta obra se distribuye con la licencia Creative Commons 
alumno tendrá experiencias de aprendizaje diferentes con la misma secuencia de actividades encadenadas. Escuchar las discusiones de los grupos es crucial para saber el nivel en el que se encuentran los alumnos.

En cuanto a la evaluación del aprendizaje del alumno, debe fundamentarse en obtener una idea general del conocimiento sobre el contenido del tema que tiene el grupo antes de iniciar el CIMA para conocer los obstáculos que deben superar los alumnos hasta alcanzar un aprendizaje duradero y de calidad. Para ello, como veremos en siguientes apartados, se utilizaron escaleras de aprendizaje.

Como se verá en el presente capítulo, la base sobre la que se cimienta este CIMA es el trabajo en equipo como motor de autoconfianza e iniciador de la motivación necesaria para el aprendizaje.

\section{Mapa de contenidos}

El mapa de contenidos elaborado se presenta en la figura 1. Como se puede observar, consta de varias partes que se corresponden con los contenidos clave que se van a tratar. Los aspectos relacionados con cada contenido clave se muestran en un color diferente.

Antes de afrontar el estudio de la Química Orgánica, los alumnos deben preguntarse cual es la relación de esta materia con la Farmacia y la profesión farmacéutica, conocer esta relación les ayudará a mantener una actitud curiosa y motivada para el aprendizaje y deberá estar presente en sus mentes durante todo el curso, por lo que se puede afirmar que es un contenido actitudinal transversal.

Por otra parte, para poder estudiar Química Orgánica, también es necesario conocer su historia (contenido que 
se encuentra enmarcado en naranja en el mapa de contenidos), se trata de un bloque de contenidos cuyo abordaje permite al alumno tomar conciencia de lo importante que es la Química Orgánica para el avance de la humanidad, así como de la Farmacia y de que cada aportación permite crecer en conocimiento, lo que supone un aprendizaje actitudinal y de valores por parte del alumno. Asimismo, estos contenidos permitirán al alumnado conocer los experimentos y observaciones clave que se han realizado a lo largo de la historia, lo que supondrá un aprendizaje de tipo procedimental y conceptual.

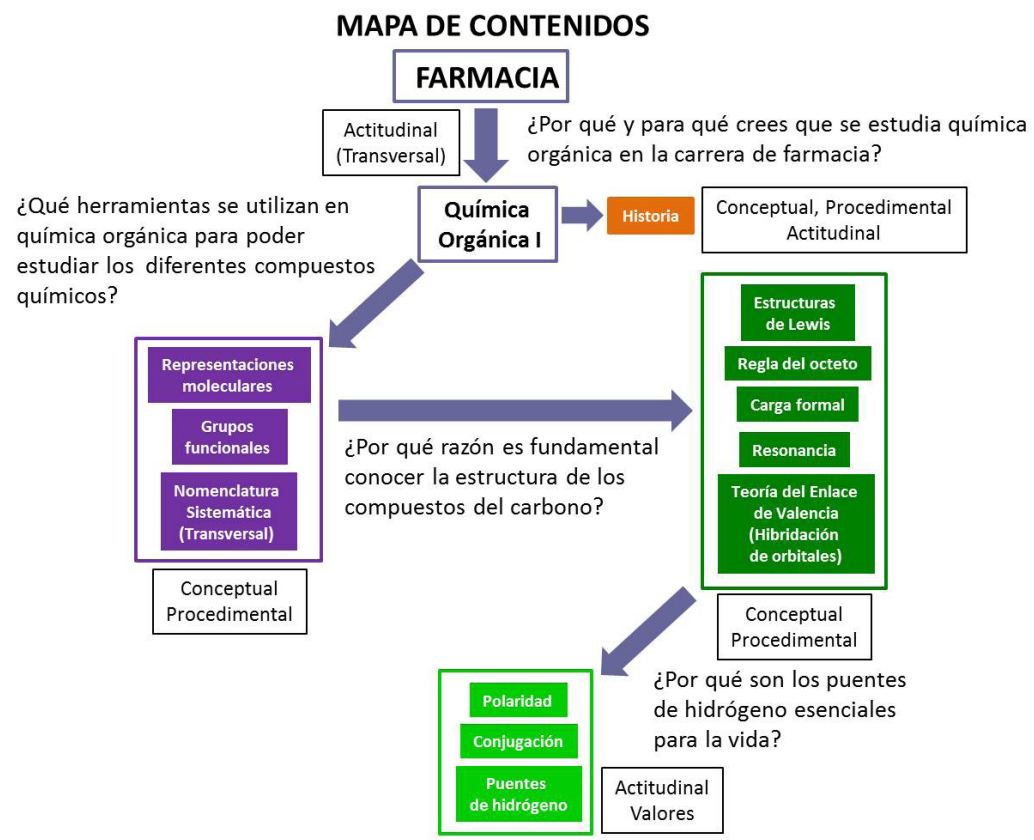

Figura 1. Mapa de contenidos y preguntas estructurantes.

El color morado se corresponde con los contenidos relacionados con la estructura química. Así, en este punto es importante que los estudiantes tengan claro que para poder estudiar la estructura, propiedades y funciones de los compuestos químicos, los científicos han elaborado una herramienta fundamental que es la de los modelos y las representaciones moleculares, por tanto, uno de los Ciclos de Mejora en el Aula (2020). Experiencias de Innovación Docente de la US 
requisitos fundamentales para el aprendizaje de la Química Orgánica es conocer y saber utilizar los distintos tipos de modelos y representaciones que existen. Solo manejando con soltura este contenido conceptual y procedimental, los estudiantes serán capaces de avanzar en su aprendizaje y así poder diferenciar cada uno de los grupos funcionales presentes en los compuestos orgánicos. Partiendo de estos conocimientos, ya se puede abordar el siguiente contenido relacionado con la estructura química: la nomenclatura. Las reglas de nomenclatura y formulación de los compuestos químicos establecidas por la IUPAC (siglas en inglés de la Unión Internacional de Química Pura y Aplicada) constituyen el lenguaje que permite a los químicos "llamar" a cada compuesto por su nombre y por lo tanto poder estudiarlos y relacionarlos. Para manejar las reglas de la IUPAC, es necesario tiempo y práctica y aunque se abordan al inicio de la asignatura, se trata de un contenido procedimental que estará presente a lo largo de todo el curso también de manera transversal.

En verde oscuro se diferencian los contenidos del tema relacionados con el enlace en los compuestos orgánicos, se trata de contenidos teóricos cuya comprensión requiere, además de un cierto conocimiento previo sobre los átomos y su configuración electrónica, familiarizarse con los símbolos que se utilizan en Química Orgánica para representar los electrones, los enlaces y las cargas, así como la adquisición progresiva de cierta capacidad de abstracción y de visión tridimensional para comprender representaciones y dibujos realizados en el plano. A medida que se van abordando estos contenidos, se va poniendo en relieve la relación de la geometría que puede presentar el carbono en los compuestos orgánicos con sus propiedades y su reactividad.

Por último, en color verde claro, se reflejan los contenidos relacionados con algunas de las propiedades de los 
compuestos orgánicos que se pueden empezar a abordar con los conocimientos que se han ido adquiriendo hasta el momento, como son la polaridad y la conjugación, que se trata de contenidos conceptuales y procedimentales y por último los puentes de hidrógeno, que por su gran importancia, ya que son fundamentales para la vida al mantener unidas las dos hebras del ADN, constituyen contenidos no solo conceptuales y procedimentales sino también actitudinales y su abordaje permite a los alumnos el aprendizaje de valores.

Estos tres grandes bloques de contenidos, están íntimamente relacionados entre sí y el aprendizaje de uno de ellos no puede ser independiente del de los demás para conocer los aspectos fundamentales del tema y su relación con la Farmacia y con el mundo que les rodea.

Todos los contenidos se abordan mediante preguntas estructurantes que también vienen reflejadas en el mapa de contenidos. Las diseñadas para este tema fueron las siguientes:

1. ¿Por qué y para qué crees que se estudia Química Orgánica en la carrera de Farmacia?

2. ¿Qué herramientas se utilizan en Química Orgánica para poder estudiar los diferentes compuestos químicos?

3. ¿Por qué razón es fundamental conocer la estructura tridimensional de los compuestos del carbono?

4. ¿Por qué los puentes de hidrógeno son esenciales para la vida?

Estas cuatro preguntas estructurantes son capaces de integrar todos los contenidos fundamentales que se tratan en este CIMA.

Ciclos de Mejora en el Aula (2020). Experiencias de Innovación Docente de la US Esta obra se distribuye con la licencia Creative Commons 


\section{Modelo metodológico y secuencia de actividades}

El modelo metodológico que se refleja en la figura 2, se repitió de la sesión 2 a la 12 del CIMA con pequeñas modificaciones referidas a los tiempos de cada actividad.

En la sesión 1, tras la presentación de la asignatura, los alumnos contestaron un cuestionario inicial que contenía las preguntas estructurantes del tema y se crearon los grupos de trabajo. En ambos casos se hizo uso de la enseñanza virtual, así, para el cuestionario se utilizó la herramienta exámenes, encuestas y bancos de preguntas y para la creación de los grupos se usó la herramienta grupos, que permite que los miembros de un mismo grupo puedan crear foros de discusión y conectarse a una sala de Bb Collaborate para trabajar juntos on line.

Una vez finalizada la primera sesión, se fueron subiendo a la enseñanza virtual una serie de actividades así como un documento a modo de guía y la bibliografía recomendada. Con este material y todo lo que tuvieran a su disposición (internet, libros, apuntes de años anteriores, etc.) debían trabajar las mismas actividades con el fin de resolver las preguntas estructurantes, primero de forma individual antes de las sesiones y posteriormente en grupos durante las sesiones, como se refleja en el modelo metodológico.

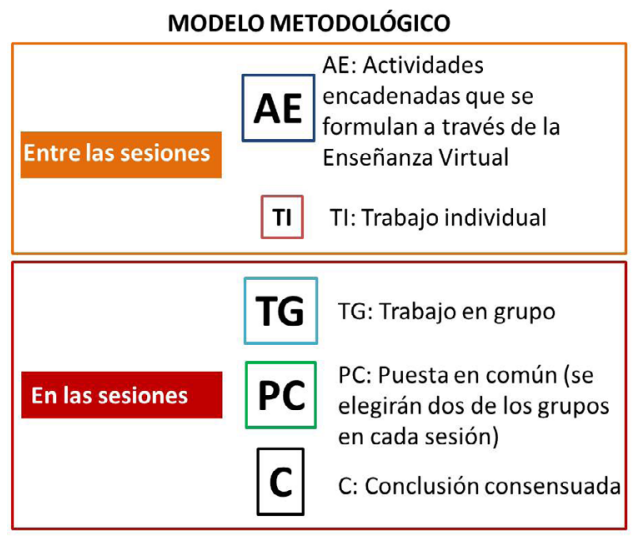

Figura 2. Modelo metodológico utilizado.

Ciclos de Mejora en el Aula (2020). Experiencias de Innovación Docente de la US Esta obra se distribuye con la licencia Creative Commons 
Las actividades, que se diseñaron para ayudar a los estudiantes a responder a las preguntas estructurantes del tema, se fueron publicando en la enseñanza virtual a través de la herramienta actividades que permite diferenciar entre entregas individuales y grupales. Todas ellas se muestran a continuación:

\section{Sesión 2: Farmacia y Química Orgánica}

1. Utilizando todos los medios a su alcance, busque la mayor información posible (estructura, actividad, efectos adversos, usos, etc.) sobre la morfina, un fármaco de origen natural, y el ibuprofeno, un fármaco de origen sintético. Elabore un documento que recoja esta información

2. Comente razonadamente el significado de la palabra fármaco y la diferencia entre un fármaco de origen natural y un fármaco de origen sintético.

Tras esta primera sesión, el siguiente contenido "Recorrido histórico de la Química Orgánica" se abordó mediante una actividad individual que los estudiantes debían entregar a la semana siguiente.

Actividad individual: Elabore una línea del tiempo en la que se recojan y se comenten los principales hitos de la Química Orgánica desde sus orígenes hasta la actualidad.

Todas las actividades propuestas hasta este punto, se diseñaron con el fin de ayudar a los estudiantes a entender por qué y para qué es importante estudiar Química Orgánica en la carrera de Farmacia, que constituye la primera pregunta estructurante y por tanto clave para la comprensión de la asignatura.

Sesión 3: Representaciones moleculares y grupos funcionales

1. Represente el 1-butanol de todas las formas posibles

2. Utilice la representación condensada y la lineal (zigzag) para dibujar todos los isómeros que tienen la 
fórmula molecular C5H12. Nómbrelos e indique qué tipo de compuestos orgánicos son.

3. En la estructura de la morfina y del ibuprofeno señale y nombre todos los grupos funcionales que poseen.

Sesión 4: Nomenclatura sistemática y formulación (Alcanos y grupos alquilo)

1. De todos los isómeros constitucionales que tienen la fórmula molecular $\mathrm{C} 8 \mathrm{H} 18$, represente en zig-zag y nombre según la IUPAC los que se nombran como derivados del hexano.

2. Dibuje utilizando la representación lineal (zig-zag) los siguientes grupos alquílicos: 1-ciclohexiletil, 2-etilhexil, 2-cilcohexiletil, bencil y fenil.

3. Nombre los siguientes grupos alquílicos e identifíquelos como grupos primarios, secundarios o terciarios:1

Sesión 5: Nomenclatura sistemática y formulación (Cicloalcanos, alquenos, alquinos y arenos)

1. ¿Cuál sería la representación lineal del ciclohexilciclohexano, isopropilciclohexano y 2-ciclopentil-2-propanol? ¿Y el nombre IUPACy la representación lineal de la siguiente estructura?:1

2. Continúe practicando la representación lineal de los compuestos orgánicos con los siguientes ejemplos: 1-Bromo-3-tercbutilciclohexeno, 1-bromo-6-ciclopropil-1-ciclohexeno y 4-alil-1-ciclopenteno. El siguiente compuesto es un alquino ramificado, represéntelo en zig-zag y nómbrelo siguiendo las normas de la IUPAC:1

3. Dibuje la estructura de los siguientes compuestos aromáticos: Estireno, ácido benzoico, 1-cloro-1-feniletano. ¿Cuál es el nombre IUPAC del siguiente compuesto?:1

Ciclos de Mejora en el Aula (2020). Experiencias de Innovación Docente de la US Esta obra se distribuye con la licencia Creative Commons 
Sesión 6: Nomenclatura sistemática y formulación. Nomenclatura común no sistemática y acrónimos

1. Rellene la siguiente tabla de compuestos que poseen una nomenclatura común no sistemática admitida por la IUPAC. Se trata de nombres que aparecerán a lo largo de toda la asignatura, así como en los libros de texto y en los artículos que se consulten.

2. Rellene la siguiente tabla de compuestos que se suelen nombrar con acrónimos. Estos acrónimos aparecerán a lo largo de toda la asignatura y al estar admitidos por la IUPAC, también van a aparecer en los libros de texto y en los artículos que se consulten.1

En este caso, para el trabajo en grupos durante la sesión 6, se realizaron Quizs a través de la herramienta Wooclap de la enseñanza virtual. Estos Quizs eran juegos de correspondencia en los que tenían que unir elementos de la columna de la izquierda con sus correspondientes elementos en la columna de la derecha.

Las actividades diseñadas para las sesiones de la 2 a la 6 pretendieron ayudar a los estudiantes a responder a la segunda de las preguntas estructurantes del tema: ¿Qué herramientas se utilizan en Química Orgánica para poder estudiar los diferentes compuestos químicos?

Sesión 7: Estructuras de Lewis, regla del octeto y carga formal

Utilizando toda la información de la que dispone, realice las siguientes actividades:

1. Represente la estructura de Lewis del dióxido de carbono, cloruro de etilo, formaldehido y ácido fórmico. Indique en el caso del cloruro de etilo y del formaldehido si la densidad de carga del carbono unido al cloro y del carbono unido al oxígeno respectivamente es positiva o negativa y explique por qué.

Ciclos de Mejora en el Aula (2020). Experiencias de Innovación Docente de la US Esta obra se distribuye con la licencia Creative Commons 
2. Complete la estructura de Lewis de las siguientes estructuras indicando todas las cargas formales y la carga neta de cada especie:1

3. Complete las siguientes estructuras de Lewis de manera que ninguno de los átomos tenga carga formal:1

Sesión 8: Resonancia

1. Represente las formas resonantes de las siguientes estructuras:1

2. Responda a las siguientes preguntas relacionadas con las fórmulas A, B y C:1

a) ¿Son formas resonantes o isómeros?

b) ¿Cuál de ellas tiene un carbono con carga formal negativa?

c) ¿Cuál es la carga neta de cada una de ellas?

d) ¿Cuál de las estructuras es más estable, A o B?

3. a) Descubra cual de las siguientes fórmulas no es un híbrido en resonancia permitido, explique por qué.1

b) Ordene las restantes fórmulas de mayor a menor contribución al híbrido en resonancia y explique las razones.

c) Utilice flechas curvadas para indicar el movimiento de electrones que conecta las tres formas resonantes.

Sesiones 9 y 10: Teoría del Enlace de Valencia (Hibridación de orbitales)

1. Mire atentamente los vídeos cuyos enlaces se muestran a continuación (enlaces a los vídeos) y exprese detalladamente los pasos a seguir para explicar los siguientes hechos:

a) Que el carbono del metano tenga hibridación sp3 y que el metano sea tetraédrico,

Ciclos de Mejora en el Aula (2020). Experiencias de Innovación Docente de la US Esta obra se distribuye con la licencia Creative Commons 

b) Que los carbonos del etileno tengan hibridación sp2 y que el etileno sea plano

2. Que los carbonos del acetileno tengan hibridación sp y que el acetileno sea lineal

3. Dibuje el metano, el etileno y el acetileno indicando los orbitales híbridos y la geometría que presentan.

4. Nombre las siguientes estructuras e indique la hibridación de los carbonos a-g señalados con flechas.1

La puesta en común y la conclusión tuvieron lugar esta vez al final de la sesión 10.

\section{Sesión 11: Polaridad y conjugación}

1. ¿Cuales de los siguientes compuestos presentan momento dipolar? Especifique su dirección.1

2. Para cada una de las siguientes moléculas con enlaces covalentes polares, indicar el extremo positivo y el negativo usando la flecha adecuada y la tabla de las electronegatividades.1

3. De cada una de las siguientes parejas de compuestos, indique el que tiene mayor momento dipolar y explique por qué, para ello utilice la flecha que indica la dirección de la polaridad del enlace.1

4. ¿Por qué es importante conocer la polaridad de los enlaces y de los compuestos orgánicos? Proporcione una explicación breve y razonada.

5. Indicar cuales de las siguientes estructuras presentan conjugación y en los casos en los que ésta exista, indicar con flechas curvadas cómo sería el movimiento de electrones.1

Las actividades propuestas en las sesiones de la 7 a la 11, iban encaminadas a hacer reflexionar a los estudiantes sobre por qué es importante conocer la estructura de los compuestos orgánicos que constituye la tercera de las preguntas estructurantes. 
Sesión 12: Puentes de hidrógeno

1. Represente puentes de hidrógeno entre el agua y el metanol

2. Explique por qué dos moléculas tan similares en peso molecular y tamaño como el etanol y el fluoroetano presentan puntos de ebullición tan diferentes (78으 y -32으 respectivamente).

3. Busque algún dibujo o hágalo usted mismo/a en el que se puedan observar los puentes de hidrógeno en el ADN.

Todas estas actividades de la sesión 12, pretendieron ayudar a que los estudiantes fueran capaces de responder a la pregunta de por qué los puentes de hidrógeno son tan importantes incluso para la vida, que es la última de las preguntas estructurantes.

Para finalizar, en esta sesión los alumnos volvieron a responder el cuestionario con las preguntas estructurantes y por último se realizó una evaluación final del CIMA.

El resumen de esta secuencia de actividades que da sentido al modelo metodológico se muestra en la figura 3. Para el trabajo en grupo, los tiempos se marcaron de forma muy estricta, así como la función que debía cumplir cada miembro del grupo: uno debía escribir las respuestas consensuadas en un documento que había que entregar después de cada sesión (se especificaron los días de entrega), otra persona debía escribir en el documento la explicación a dichas respuestas y otro actuar de portavoz del grupo durante la puesta en común. Todas estas funciones fueron rotando de modo que cada miembro al final del CIMA había pasado por todos los roles.

Ciclos de Mejora en el Aula (2020). Experiencias de Innovación Docente de la US Esta obra se distribuye con la licencia Creative Commons 
SECUENCIA DE ACTIVIDADES

\begin{tabular}{|c|c|c|}
\hline Etapa & Actividad & Tiempo \\
\hline 1: AE & $\begin{array}{c}\text { A través de la plataforma virtual se plantean } \\
\text { las actividades encadenadas }\end{array}$ & $\begin{array}{l}\text { Antes de las sesiones } \\
\qquad \text { del CIMA }\end{array}$ \\
\hline 2: $\mathrm{TI}$ & Trabajar individualmente las actividades & $\begin{array}{l}\text { iempo comprendido } \\
\text { ntre que se suben las } \\
\text { actividades a la EV y } \\
\text { cada sesión }\end{array}$ \\
\hline 3: TG & $\begin{array}{l}\text { Los grupos trabajan sobre las preguntas que } \\
\text { previamente han trabajado de forma individual }\end{array}$ & $35^{\prime}$ aprox. \\
\hline 4: PC & $\begin{array}{c}\text { Se eligen dos grupos para contrastar las } \\
\text { respuestas, los demás grupos se posicionan } \\
\text { y/o aportan alguna variante }\end{array}$ & $15^{\prime}$ aprox. \\
\hline 5: C & $\begin{array}{l}\text { Alcanzar un consenso entre todos los grupos. En } \\
\text { este punto, si es necesario, interviene el docente } \\
\text { remarcando los puntos más importantes. }\end{array}$ & 10' aprox. \\
\hline
\end{tabular}

Figura 3. Secuencia de actividades del CIMA

\section{Aplicación del CIMA}

\section{Relato resumido de las sesiones}

Este relato está dividido en tres semanas, ya que las doce sesiones del CIMA se distribuían en cuatro horas cada semana. La primera semana de implementación del nuevo sistema de enseñanza-aprendizaje, debido a que coincidió exactamente con la primera semana del curso, resultó compleja, pues los alumnos no solo se tenían que adaptar a la nueva metodología sino que también, al ser una asignatura del primer curso, tenían que adaptarse a todo lo demás: el paso de Secundaria a la Universidad, los nuevos compañeros de clase, el nuevo centro de estudios, etc., con el agravante de la reciente implementación del sistema bimodal de enseñanza en la Universidad de Sevilla

Ciclos de Mejora en el Aula (2020). Experiencias de Innovación Docente de la US Esta obra se distribuye con la licencia Creative Commons 
debido a la pandemia de la COVID-19. Si, dejando aparte todas estas novedades, nos centramos solo en el análisis de la primera semana con respecto al nuevo sistema de enseñanza-aprendizaje propuesto por este CIMA, lo más importante que puedo destacar es que todo proceso de cambio requiere un período de adaptación y en este caso, se evidenció en la primera semana. Al tratarse de un CIMA en el que se utilizaban un gran número de herramientas proporcionadas por la Enseñanza Virtual de la US, durante la primera semana lo más complejo, tanto para los estudiantes como para mí, fue adaptarnos al funcionamiento de todas estas nuevas herramientas.

Durante la segunda semana, las sesiones transcurrieron con mucha mayor facilidad que en la semana anterior, debido a que ya nos habíamos adaptado tanto a la metodología, como a las herramientas. Fue un cambio de gran envergadura el que pudimos experimentar durante esta semana. Desaparecieron los problemas técnicos que ocupaban gran parte de la hora de las sesiones durante la primera semana y además, utilicé por primera vez una herramienta que tuvo muy buena acogida. Esta herramienta consistió en grabar y compartir con los alumnos a través de la Enseñanza Virtual una serie de vídeos cortos en los que hacía hincapié sobre aspectos que consideraba suficientemente importantes para el aprovechamiento de las sesiones y de la asignatura.

Con respecto a la tercera semana, lo más destacado fue sin duda la gran implicación del alumnado con su propio trabajo, con sus grupos de trabajo y con la puesta en común de las actividades. Para mí fue gratamente sorprendente.

Ciclos de Mejora en el Aula (2020). Experiencias de Innovación Docente de la US Esta obra se distribuye con la licencia Creative Commons 


\section{Evaluación del aprendizaje de los estudiantes}

Como ya se indicó en apartados anteriores, con el propósito de evaluar el aprendizaje de los estudiantes durante el CIMA, se utilizaron escaleras iniciales y finales de aprendizaje. Las escaleras se elaboraron pasando el cuestionario a los alumnos con las preguntas o problemas estructurantes del tema, antes de empezar el ciclo de mejora y al finalizarlo. Las respuestas de los alumnos se clasificaron de menor a mayor adecuación a la respuesta prototipo ideal, cada respuesta se hizo corresponder con un nivel de aprendizaje y la distancia entre los niveles dependió de la complejidad del obstáculo que había que superar para pasar a un nivel superior. Una vez elaborada la escalera, se calculó el porcentaje de estudiantes que había en cada nivel. De esta forma, se tuvo una idea general del conocimiento sobre el tema que tenía el grupo así como cada alumno de forma individual antes de iniciar el ciclo de mejora y al finalizarlo. La escalera inicial, permitió además conocer los obstáculos que tenían los estudiantes para pasar a niveles más elevados de conocimiento. Por último, la evolución del resultado de la escalera final con respecto a la inicial, fue utilizada como uno de los procedimientos de evaluación de los alumnos del curso así como las actividades encadenadas entregadas, tanto la individual (Línea del tiempo) como las grupales.

En la figura 4, se muestran las escaleras de aprendizaje para todas las preguntas, los patrones de respuestas que constituyeron los distintos niveles, los posibles obstáculos para ascender de nivel y los porcentajes de alumnos que se situaron en cada nivel tanto al inicio del ciclo como al final.

Ciclos de Mejora en el Aula (2020). Experiencias de Innovación Docente de la US Esta obra se distribuye con la licencia Creative Commons 


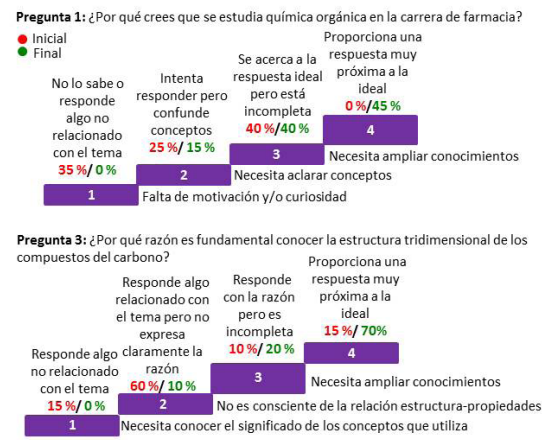

Pregunta 2: ¿Qué herramientas se usan en química orgánica para poder estudia os diferentes compuestos químicos?? Proporciona una respuesta muy
próxima a la

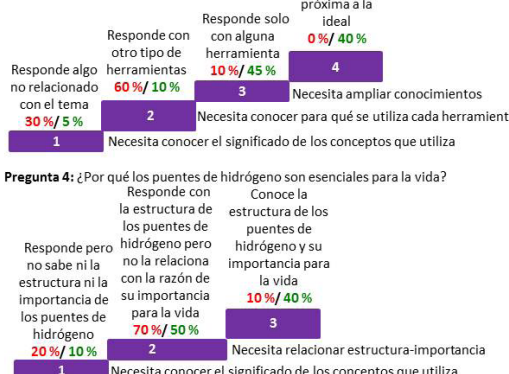

Figura 4. Escaleras de aprendizaje

Comparando los porcentajes de las escaleras iniciales y finales de aprendizaje, se puede decir que en general los alumnos con los que se aplicó esta metodología fueron capaces de superar la mayoría de los obstáculos que les impedían adquirir niveles elevados de conocimiento relacionados con los temas de estudio.

La escalera de aprendizaje elaborada con la primera pregunta del cuestionario, muestra que el 35\% de los alumnos que realizaron el cuestionario inicial no sabía por qué era necesario estudiar Química Orgánica en la carrera de Farmacia o respondió algo no relacionado con el tema (nivel 1), lo que denotaba que el principal obstáculo para el aprendizaje de estos alumnos era la falta de motivación y de curiosidad. Por su parte, el $25 \%$ de ellos, intentó responder pero confundía conceptos, en este caso, el obstáculo era la necesidad de aclarar conceptos. Ascendiendo a un nivel superior, nos encontramos con que el $40 \%$ de la clase, es decir, la mayoría de los alumnos, dio una respuesta cercana a la ideal pero incompleta, el obstáculo en este caso sería la necesidad de este grupo de alumnos de ampliar conocimientos con respecto a los que ya tenían. En la escalera final, lo más significativo que se observó fue que el número de alumnos en el nivel 3 seguía siendo del $40 \%$ y el de alumnos en el nivel 4 pasó del 0 al $45 \%$, de lo que se dedujo que la mayoría de los estudiantes superó la mayoría de los obstáculos de aprendizaje. 
La escalera de aprendizaje elaborada con la pregunta 2 del cuestionario, que informaba sobre la idea que tenían los estudiantes sobre las herramientas que se utilizan en Química Orgánica para estudiar los compuestos químicos, mostró que el $30 \%$ de los alumnos que realizaron el cuestionario inicial escribió algo no relacionado con el tema, por lo que estas personas necesitaban conocer el significado de los conceptos que utilizaban para hacerlo con propiedad. En el siguiente nivel de aprendizaje se encontró el $60 \%$ de la clase, se trataba de estudiantes que sabían de la existencia de algunas herramientas pero no precisamente las que se utilizan para estudiar de un modo teórico las estructuras químicas, por lo que estos estudiantes necesitaban identificar cada herramienta con su finalidad. El 10 \% escribió sólo algunas herramientas, por lo que dicho porcentaje de alumnos necesitaba conocer todas las demás herramientas. En la escalera final, se observó que esta vez el porcentaje de alumnos en el nivel 1 fue del 5\%, sólo el 10\% se encontraba ahora en el nivel 2, el 45\% de los alumnos estaba en el nivel 3 y el $40 \%$ de todos, había superado todos los obstáculos de aprendizaje anteriormente comentados y se encontraba en el máximo nivel.

La escalera de aprendizaje elaborada con la pregunta 3 del cuestionario, relacionada con la idea que tenían los estudiantes sobre la relación entre la estructura y las propiedades de los compuestos químicos, mostró que la mayoría de la clase se situó en el segundo nivel de conocimiento, respondiendo algo relacionado con el tema pero no expresando claramente la relación estructura tridimensional-propiedades debido a que no es consciente de la existencia de esta relación ya que necesita ampliar conocimientos. Sin embargo, en la escalera final, el 70\% de los estudiantes se situó esta vez en el nivel superior, superando gracias a la aplicación del CIMA todos los obstáculos para el aprendizaje.

Ciclos de Mejora en el Aula (2020). Experiencias de Innovación Docente de la US Esta obra se distribuye con la licencia Creative Commons 
La escalera de aprendizaje elaborada con la pregunta 4 del cuestionario inicial, que informaba sobre la idea que tenían los estudiantes sobre los puentes de hidrógeno y su relación con la vida, mostró que la gran mayoría de la clase (70\%), conocía la estructura de los puentes de hidrógeno y su naturaleza, sin embargo no sabía la relación entre esta estructura y su importancia para la vida, lo que constituía el principal obstáculo para su aprendizaje. Después de la aplicación del CIMA, en este nivel se situó un porcentaje menor de estudiantes (el 50\%) mientras que el 40\% de los estudiantes ya era capaz de relacionar la estructura de los puentes de hidrógeno con su importancia para la vida.

Por último, cada uno de los informes entregados por los estudiantes después de cada actividad, se corrigió en base a una rúbrica sencilla que permitió cuantificar con una nota la realización y entrega de las actividades, esta nota, se sumará a la calificación final de la asignatura y tendrá un valor máximo de dos puntos.

\section{Evaluación del CIMA}

En primer lugar, resulta obligado destacar que, aunque la autora había realizado con anterioridad dos CIMA, presentados en las IV y VI Jornadas de Formación e Innovación Docente de la US (2018 y 2019), esta era la primera vez que ponía en práctica uno en la asignatura de Química Orgánica I y en un sistema de enseñanza semi-presencial con la incorporación de la cantidad de herramientas virtuales que ello conlleva. A pesar de ello, el resultado del CIMA puede considerarse satisfactorio. Sin embargo, cabe destacar que aunque el mapa de contenidos se ha podido trabajar en su totalidad y la secuencia de actividades y el modelo metodológico diseñados al inicio del CIMA prácticamente se han cumplido, sería conveniente revisar algunos aspectos del CIMA como por ejemplo incluir la realización de juegos Ciclos de Mejora en el Aula (2020). Experiencias de Innovación Docente de la US
Esta obra se distribuye con la licencia Creative Commons 
en más sesiones. Por otro lado, en futuras aplicaciones del CIMA, teniendo en cuenta la complejidad del tema de las sesiones 8 y 11, también sería conveniente que los contenidos de las mismas se pudieran tratar en tres sesiones en lugar de en dos.

Por otro lado, las escaleras de aprendizaje han resultado de gran utilidad para evaluar el diseño ya que mostraron la evolución del grupo y de cada alumno durante el desarrollo del CIMA, de modo que al finalizar, se pudo conocer el grado en que se habían alcanzado los objetivos de aprendizaje. Así, aunque no se alcanzaron al cien por cien los objetivos en el sentido de que no todos los estudiantes se localizaran en el nivel más elevado de cada escalera, en general se produjo una evolución positiva prácticamente de todos ellos.

Por último, los estudiantes que participaron en esta experiencia de enseñanza-aprendizaje tuvieron la oportunidad de evaluarla cuantitativamente calificándola con un valor numérico que iba de 0 a 5 en relación al modo en que el nuevo modelo docente les había sido útil para trabajar, estudiar y comprender mejor la materia objeto de estudio. Así, los valores numéricos se pueden corresponder con las siguientes afirmaciones:

0 : No me ha sido útil

1: Me ha sido útil sólo para trabajar, pero no para estudiar ni comprender mejor la materia objeto de estudio

2: Me ha sido útil para trabajar y estudiar, pero no para comprender mejor la materia objeto de estudio

3: Me ha sido útil para trabajar, estudiar y comprender algo la materia objeto de estudio

4: Me ha sido útil para trabajar, estudiar y comprender mejor la materia objeto de estudio

5: Me ha sido útil para trabajar, estudiar y comprender mucho mejor la materia objeto de estudio. 
Los resultados de esta valoración se muestran en la figura 5.

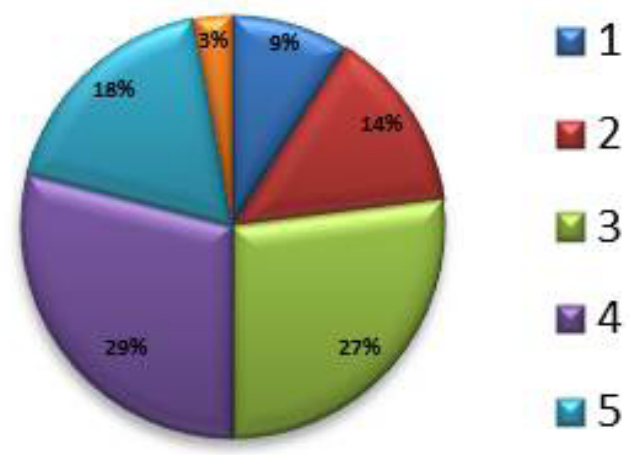

Figura 5. Evaluación cuantitativa del CIMA por los estudiantes

Como se puede observar en la figura 5 , el $74 \%$ de los estudiantes valoró positivamente el CIMA en cuanto a su utilidad a la hora de trabajar, estudiar y comprender el contenido del tema, de ese $74 \%$, el $27 \%$ consideró que es una metodología útil para trabajar, estudiar y comprender algo el tema (valoración = 3), el 29\% consideró que es útil para trabajar, estudiar y comprenderlo mejor (valoración = 4) y el $18 \%$ consideró que es útil para trabajar, estudiar y comprenderlo mucho mejor (valoración $=5$ ). El resto de estudiantes consideró que el método le había servido poco, aun así, ninguno le dio una valoración de 0 y sólo un 3\% optó por no dar una valoración a la experiencia.

Además de esta evaluación cuantitativa, los estudiantes pudieron responder brevemente a las siguientes preguntas a modo de evaluación personal y cualitativa que permitirá introducir mejoras en posteriores aplicaciones del CIMA:

1. ¿Qué eliminarías de esta forma de trabajar?

2. ¿Qué incorporarías?

3. ¿Qué mejorarías? ¿Cómo?

Ciclos de Mejora en el Aula (2020). Experiencias de Innovación Docente de la US Esta obra se distribuye con la licencia Creative Commons 
Así, la mayoría de los estudiantes consideraron que no eliminarían nada, sin embargo, incorporarían alguna explicación por parte del docente antes de abordar la realización de las actividades y en cuanto a las posibles mejoras, la mayoría incorporaría la realización de más vídeos de apoyo a las actividades y tener más tiempo para asimilar los contenidos.

Los principios didácticos que se han aplicado en este CIMA, han dado muy buenos resultados, por lo que es mi intención mantenerlos en próximos CIMA. Se trata de formular preguntas que recojan los principales contenidos de cada tema y plantear actividades para abordarlos. Estas actividades se han de trabajar primero de forma individual y después en grupo y deben servir a los estudiantes como guía que les permita, además de resolver las preguntas, motivarse a descubrir y pensar por ellos mismos, para encontrar sus propios resultados y posteriormente contrastarlos con los de los demás grupos y con el docente y, de esta forma, generar el aprendizaje. Así mismo, tengo el propósito de estar atenta a los grupos, observar su modo de trabajar y acompañarlos en su experiencia, para ir conociéndolos mejor en cuanto a su relación con los demás miembros del grupo, con el resto de la clase y con la asignatura, lo que me irá guiando en el diseño de las siguientes actividades. Mantendré el uso de muchas de las herramientas de la Enseñanza Virtual de la Universidad de Sevilla ya utilizadas e incorporaré otras nuevas como Teams ${ }^{\circledR}$ para abrir un foro de la asignatura, donde el grupo pueda discutir cualquier actividad o duda que se le plantee. Por último, con todo este material, elaboraré más vídeos a modo de píldoras audiovisuales que les sirvan de ayuda.

1 Por cuestiones de espacio, en esta publicación no se han insertado ni las figuras ni las tablas de las actividades.

Ciclos de Mejora en el Aula (2020). Experiencias de Innovación Docente de la US Esta obra se distribuye con la licencia Creative Commons 
Palabras clave: Química Orgánica I, Grado en Farmacia, docencia universitaria, experimentación docente universitaria, estudio y comprensión de la estructura y el enlace.

Key words: Organic Chemisty I, Degree in Pharmacy, University teaching, University teaching research, Study and comprehension of structure and chemical bond-

\section{Referencias bibliográficas}

Bain, K. (2004). Lo que hacen los mejores profesores universitarios. Valencia: Publicaciones de la Universidad de Valencia.

De-Alba-Fernández, N. y Porlán, R. (2020). Docentes universitarios. Una formación centrada en la práctica. Madrid: Ediciones Morata.

Finkel, D. (2008). Dar clases con la boca cerrada. Publicaciones de la Valencia: Universidad de Valencia.

Porlán, R. (Coord.) (2017). Enseñanza universitaria. Cómo Mejorarla. Madrid: Morata.

Valdivia, V. (2017). Un modelo docente innovador para la interpretación de la tabla periódica. IV Jornadas de Formación e Innovación Docente de la US. Sevilla: Editorial de la Universidad de Sevilla.

Valdivia, V. (2019). Ciclo de mejora en el aula para el estudio del átomo en primero del grado en educación primaria. En E. Navarro-Medina y R. Porlán (Coord.), Ciclos de mejora en el aula. Año 2019. Experiencias de innovación docente de la Universidad de Sevilla (pp. 819-843). Sevilla: Editorial de la Universidad de Sevilla

Ciclos de Mejora en el Aula (2020). Experiencias de Innovación Docente de la US Esta obra se distribuye con la licencia Creative Commons 